\title{
Hardware-in-the-Loop-Simulation of a Building Energy and Control System to Investigate Circulating Pump Control Using Modelica
}

\author{
Georg Ferdinand Schneider ${ }^{1} \quad$ Jens Oppermann ${ }^{2} \quad$ Ana Constantin $^{3} \quad$ Rita Streblow $^{3}$ Dirk Müller ${ }^{3}$ \\ ${ }^{1}$ Fraunhofer Institute for Building Physics, Systems Integration Group, Fürther Straße 250, 90429 Nürnberg, \\ Germany, georg.schneider@ibp. fraunhofer.de \\ ${ }^{2}$ WILO SE, Group Research and Technology, Nortkirchenstraße 100, 44263 Dortmund, Germany, \\ Jens. Oppermann@wilo.com \\ ${ }^{3}$ RWTH Aachen University, Institute for Energy Efficient Buildings and Indoor Climate, Matthieustraße 10, \\ 52074 Aachen, Germany, \{aconstantin, rstreblow, dmueller\} @eonerc.rwth-aachen. de
}

\begin{abstract}
This paper presents an application of the hardware-inthe-loop-method to a building energy and control system. We focus on investigating the interaction of a real circulating pump with the hydronic network of a virtual building energy and control system. For real-time simulation the building envelope is modelled using the Modelica-based library AixLib. With the presented setup model-based designed control algorithms are tested directly on real hardware. The performance of the presented and implemented setup is evaluated by comparing simulated results with experimental hardware-in-the-loopsimulation data. The main focus of this work is to evaluate the application of the method towards bridging the gap between model-based design and commissioning of energy efficient control for heating ventilation and air conditioning (HVAC) components.
\end{abstract}

Keywords: Hardware-in-the-loop (HIL); Building Energy and Control System; Model-Based Design

\section{Introduction}

There is an ongoing research effort towards developing tools and processes to improve the design of building energy and control systems (BECSs) in order to reduce the environmental impact of existing and future buildings while improving their indoor comfort (Wetter, 2011a). With the ongoing reduction of the energy demand for heating and cooling of buildings, e.g. better insulation, research is focussing on increasing the energy efficiency of auxiliary HVAC equipment such as circulating pumps. Additional pressure is put on equipment manufacturers by the European Union by introducing mandatory eco-design requirements for energy-using products (EU, 2009).
To achieve these efficiency goals is an inherently difficult task as BECS typically are comprised out of heterogeneous components which have to be regarded simultaneously on different temporal and spatial scales (Wetter, 2011a). An approach to design control algorithms for components of these systems in an integrated way is the application of model-based design methods. Equationbased object-oriented modelling languages, e.g. Modelica, allow modelling these systems consistently (Wetter, 2011a). In Modelica ready-to-use libraries exist to perform whole building energy simulation for model-based design (e.g. Constantin et al., 2014; De Coninck et al., 2014; Wetter et al., 2014).

However, difficulties occurring during the transition process from design to commissioning stage of model-based control algorithms remain unsolved. The transition process can be time and cost consuming and a feedback of newly generated information to the design process is seldom possible.

Increasing circulating pump efficiency is mainly driven by implementing control algorithms into the application controller of pumps, which consider the interaction between the pump and its surrounding BECS. For the case of control algorithms for circulating pumps we observed the following obstacles:

- An error-prone reimplementation of the designed control algorithms is necessary at each iteration, as operating systems and programming languages differ between simulation environments and real hardware application controllers;

- Testing of control algorithms for circulating pumps requires detailed measurements of pump prototypes in test-buildings over time periods of days and weeks.

Hardware-in-the-loop-(HIL-) simulation is a method to bridge the gap between simulation and real hardware 
by coupling simulated and real parts of a system. The HIL-method is well-known in science and technology and several applications based on Modelica are reported, e.g. in automotive, food and microcontroller industries (Winkler and Gühmann, 2006; Gäfvert et al., 2008; Bonvini et al., 2009). In the buildings industry the HILmethod is frequently used for testing and commissioning of building control systems (e.g. Xu et al., 2004). Chen et al. (2012) present a multi-domain test setup to investigate building control and HVAC-systems with simulated boundary conditions. Applying the HIL-method to a circulating pump in the buildings domain is, to the best of our knowledge, a novelty.

The contribution aspect of this paper is the presentation of a successful application of the HIL-method to a BECS to investigate control algorithms for HVAC components. By this for the application of a circulating pump,

- model-based designed algorithms may be tested directly on real hardware without reimplementation and

- different building types can be examined on a single hardware setup by using different models.

After the introduction we present a brief description of the HIL-method in section 2. A HIL-concept for a BECS is presented in section 3. Finally, in section 4 we evaluate the performance of an implementation of the HILconcept by a comparison of measured data and simulated results.

\section{Methodology}

This section gives a brief overview of the HIL-method. In HIL-simulation real hardware is coupled to virtual components of a system which are simulated in real-time (Maclay, 1997). The loop comprises of providing the measured behaviour of real components as boundary conditions to virtual models of a system and emulating the simulated reaction at every time step to the hardware components.

The HIL-method offers several advantages in its application (Maclay, 1997). Despite the decreased development times in today's development cycles it is necessary to keep the established high standards in safety and quality of products and processes. HIL supports and simplifies research and development processes of complex systems by giving the possibility to test components of heterogeneous systems without danger for humans, environment or plants by malfunctioning or unexpected behaviour (Maclay, 1997). HIL-simulation often is easily applicable as models available from model-based development processes may be reused for HIL-simulation with minor changes. Nevertheless the accuracy of the used models has to be carefully assessed for a successful application of the method. When developing an application of the method it is necessary to evaluate the dynamic performance of the designed emulation facilities to ensure a proper feedback of the simulated behaviour to the real components.

A HIL-system can be divided into three parts: simulation, emulation and hardware level (Chen et al., 2012). At the simulation level models of the virtual components are provided and simulated in real-time. The emulation level actually enables the coupling of real and virtual system components. It includes devices and facilities to measure the hardware behaviour and to emulate the simulated behaviour of the virtual components. A real-time capable data interface is necessary to exchange data between the data acquisition devices which measure the physical quantities and the simulation environment at run time. At the hardware level real components of a complex system are installed in an emulator.

\section{Application of HIL-Method}

In this section we demonstrate the application of the HIL-method to a BECS to investigate control algorithms for circulating pumps. The details and a real setup of a HIL-concept for a circulating pump are presented. With the proposed HIL-setup it is possible to examine the interaction of a real circulating pump in a hydraulic network of a virtual BECS. In our specific case both the model of the BECS and the algorithms for circulating pump control are implemented in one simulation environment. The coupling of the simulation environment and the hardware allows to directly execute the control algorithms on a real pump. A scheme of the realized HILconcept is presented in Figure 1.

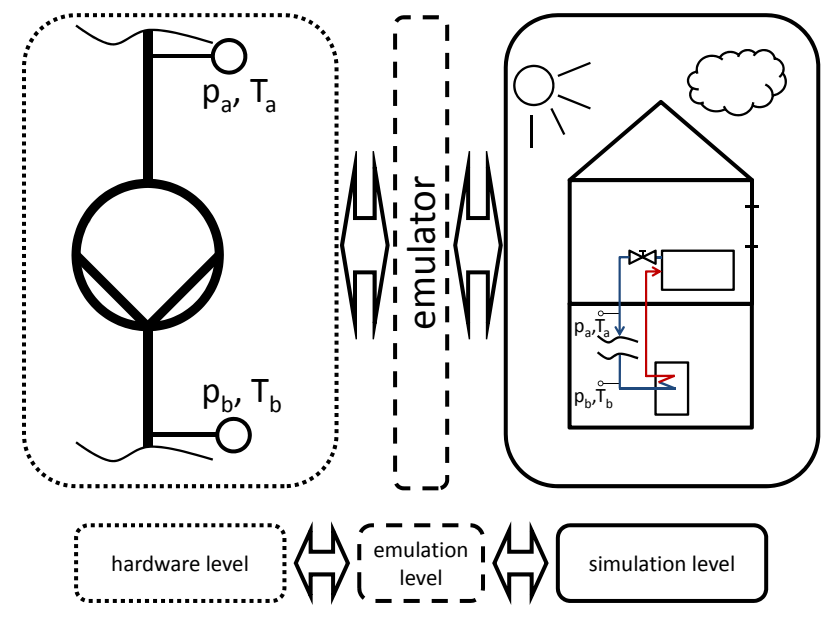

Figure 1. Scheme of the developed HIL-concept of a real pump in a virtual BECS. The boundary conditions pressure $\mathrm{p}_{\mathrm{a}, \mathrm{b}}$ and temperature $\mathrm{T}_{\mathrm{a}, \mathrm{b}}$ up- and downstream of the pump are exchanged via a data interface (arrows).

We divide the HIL-system in a hardware, emulation and simulation level. At the hardware level a real pump is 
installed in a hydraulic circuit at a testbench. The boundary conditions of the pump are characterized by pressure $\mathrm{p}_{\mathrm{a}, \mathrm{b}}$ and the temperature $\mathrm{T}_{\mathrm{a}, \mathrm{b}}$ up- and downstream of the pump. The downstream boundary conditions are measured and given as input to a BECS model at the simulation level. The upstream values are calculated by a real-time simulation of the BECS and are emulated at the emulation level to the real pump.

The following sections describe the simulation, emulation and hardware level of the proposed HIL-concept in detail.

\subsection{Simulation Level}

We choose the Modelica-based library AixLib to model one and multiple family dwellings for simulation in Dymola (Dymola, 2013). The library is open source and accessible from an online repository (https://github.com/RWTH-EBC/AixLib). We utilize ready-to-use models of the library to describe the building envelope. To model the HVAC and control system we use in this application models from the library described in Müller and Hosseini Badakhshani (2010).

The full details of the used models is not in the scope of this paper. For a detailed description please refer to the mentioned references. The main modelled aspects are:

- Each room model has one air node modelled as a single, perfectly mixed air volume;

- The air volume exchanges heat to its enclosures by convective, conductive and radiative heat transfer;

- A wall model consists of layers with different physical properties each; transient heat conduction through each layer is approximated using a one dimensional approach;

- The model of the heating system consists of radiators with thermostatic valves for each room, pipes, a boiler with night setback and an expansion tank;

- A weather file providing weather data of a typical reference year (TRY) for locations within Germany (DWD, 2013);

- User occupancy, ventilation and infiltration are considered.

Figure 2 illustrates schematically the structure and components of the modelled BECS of a one family dwelling. The models of AixLib are currently under evaluation by using a set of standardized tests provided by the American Society of Heating, Refrigerating and AirConditioning Engineers to validate building simulation models (ASHRAE, 2004). Additionally, we statically validated the building model by comparing the simulated results with results of the design heat load calculated according to EN 12831 (CEN, 2003) using the commercial tool SOLAR-COMPUTER (Solar-Computer, 2012). For the HIL-system we adapt models developed for

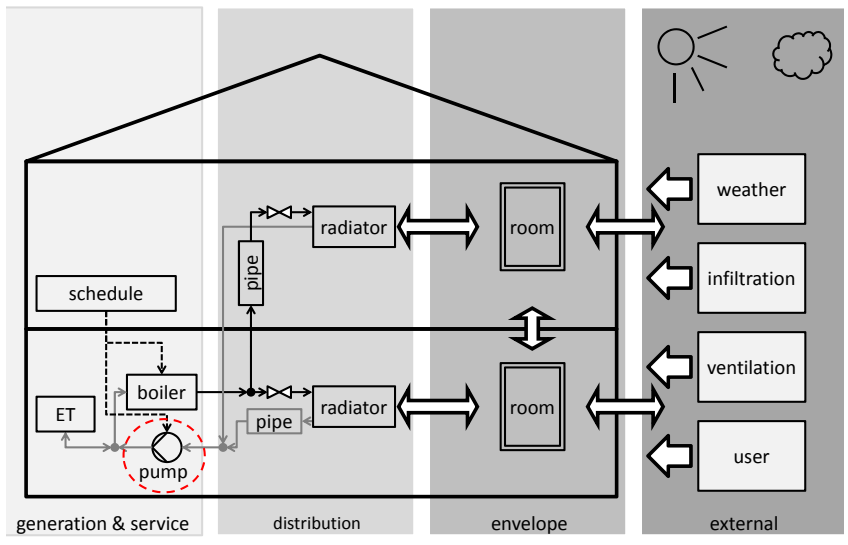

Figure 2. Scheme of the building model. Pump model (dashed, red) is substituted by two boundary conditions with inputs for the case of HIL-simulation (ET - expansion tank).

model-based design of control algorithms for circulating pumps by modifying them for HIL purposes. To clarify the model structure the top level of the simulation model is presented in a scheme in Figure 3. It comprises of a building envelope and HVAC system model and the weather input. Instead of a pump model two hydraulic boundary conditions (fluid source, fluid sink) are implemented. Together with the building models a TCP\IPbased data interface is implemented and simulated as well as the pump control algorithms. Via the data interface the value for the current pressure difference $\Delta \mathrm{p}$, the current volume flow rate of the hydraulic circuit $\mathrm{Q}$, the current set point of the pump $\mathrm{y}_{\text {pump }}$ and the set point for the motor-driven valve $\mathrm{y}_{\text {valve }}$ are exchanged at runtime. The number of equations of the resulting model is typically in the range of ten thousand.

To simulate the described models in real-time we use Dymola with default settings and its capability to synchronize with an external Dynamic Data Exchange (DDE) server for (soft) real-time simulation.

\subsection{Emulation Level}

The emulation system acts as an interface between the real hardware and the virtual components. It performs two tasks: first, it offers facilities to measure physical quantities and transmit the values to the simulation environment; second, it has the capability to convert the simulated values back into real physical quantities (Chen et al., 2012).

In this section we present a solution to emulate the hydraulic boundary conditions and a data interface which couples the LabVIEW-based testbench software (NI, 2015) and Dymola. A scheme of the system is displayed in Figure 4. We assume negligible temperature drop across the pump and therefore the facilities for the temperature emulation are not discussed here. 


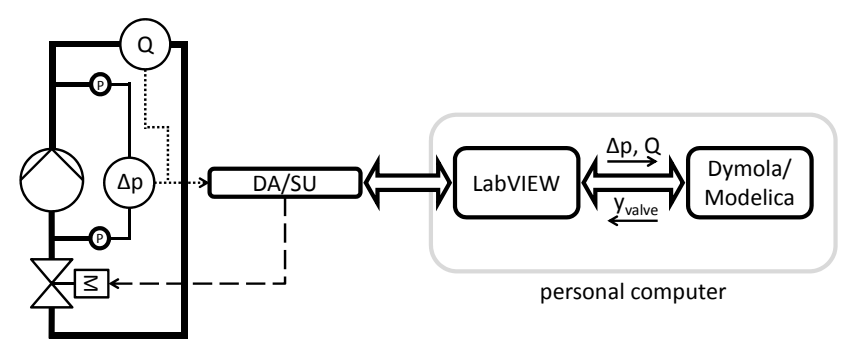

Figure 4. Scheme of the testbench setup (DA/SU - data acquisition/ switch unit). Data exchange between LabVIEW and Dymola/Modelica via a TCPIIP-based interface, $\Delta \mathrm{p}$ - pressure difference, $\mathrm{Q}$ - volume flow rate in hydraulic circuit, $\mathrm{y}_{\mathrm{valve}}-$ set point motor-driven valve.

\section{Emulation of Hydraulic Boundary Conditions:}

To establish a loop the pressure difference $\Delta \mathrm{p}$ across the pump is measured for one time step by a differential pressure sensor with precision of $1 \%$ of the measured value and is given to the simulation as input. Then the model is simulated for this time step and the corresponding total volume flow rate $\mathrm{Q}_{\text {set }}$ of the building is calculated. This volume flow rate needs to be converted into a physical quantity by the testbench.

To perform this task we measure the volume flow rate $\mathrm{Q}$ in the hydraulic circuit of the testbench by a magnetic flow meter with a precision of $0.5 \%$ of the measured value and a response time of $0.125 \mathrm{~s}$. We implement a feedback control with the simulated total volume flow rate as set point. A PI-controller with limited output implemented from the Modelica Standard Library is used to control the volume flow rate $\mathrm{Q}$ through an adjustable, motor-driven valve with a nominal stroke of $5.5 \mathrm{~mm}$, a resolution of 1:100 and a positioning speed of $13.6 \mathrm{~mm} / \mathrm{s}$.

The dynamic performance of the described volume flow rate control system can be assessed from results of an exemplary step response presented in Figure 5. Measurements are performed at a sample rate of $1 \mathrm{~s}$. From the results we measure a static deviation of $\pm 3.5 \mathrm{lh}$ and a settling time of $200 \mathrm{~s}$.

We assess the dynamics and the precision of the system to be sufficient for the considered application. Time constants in thermal systems of buildings are in the range of 1000 seconds which is one magnitude larger than the determined settling time.

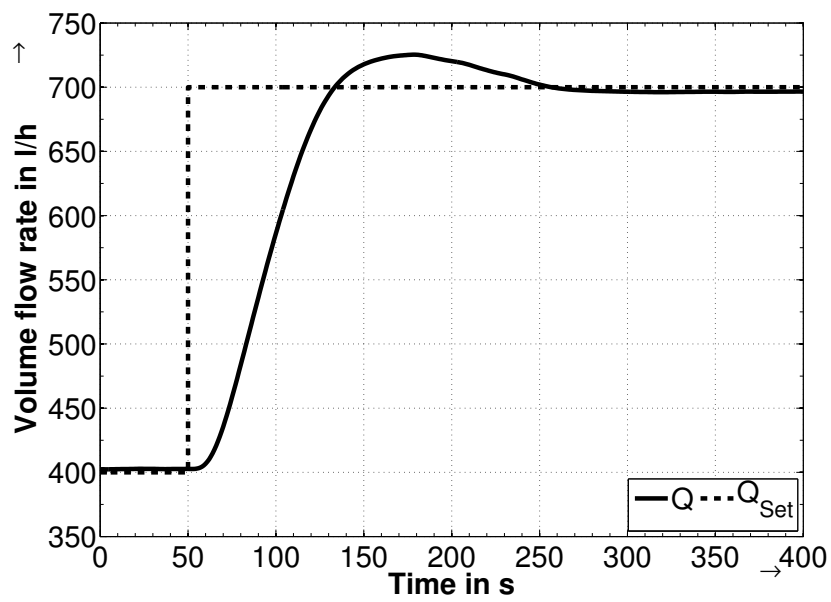

Figure 5. Step response of volume flow rate control system, $\mathrm{Q}_{\text {set }}$ - Set point of PI-control, Q - measured volume flow rate in hydraulic system, sampling rate: $1 \mathrm{~s}$.

\section{Socket-Based Data Interface:}

A prerequisite of the development of the HIL-testbench is the usage of a proprietary, LabVIEW-based data interface to enable access to the low-level pump control with the possibility to execute model-based designed control algorithms without any reimplementation. A disadvantage of this platform at the point of implementation is that no ready-to-use data interface to exchange data at run time to the simulation environment was available.

We followed a straightforward approach and implemented a real-time capable socket-based data interface to enable data exchange with the simulation environment at run time. The data interface is based on the Winsock API (Microsoft, 2015) which allows to exchange character strings via network sockets. The interface is implemented in $\mathrm{C}$ and uses a set of functions to exchange character strings using the TCP/IP protocol. To include the additional $C$ code we use the external $C$ construct of

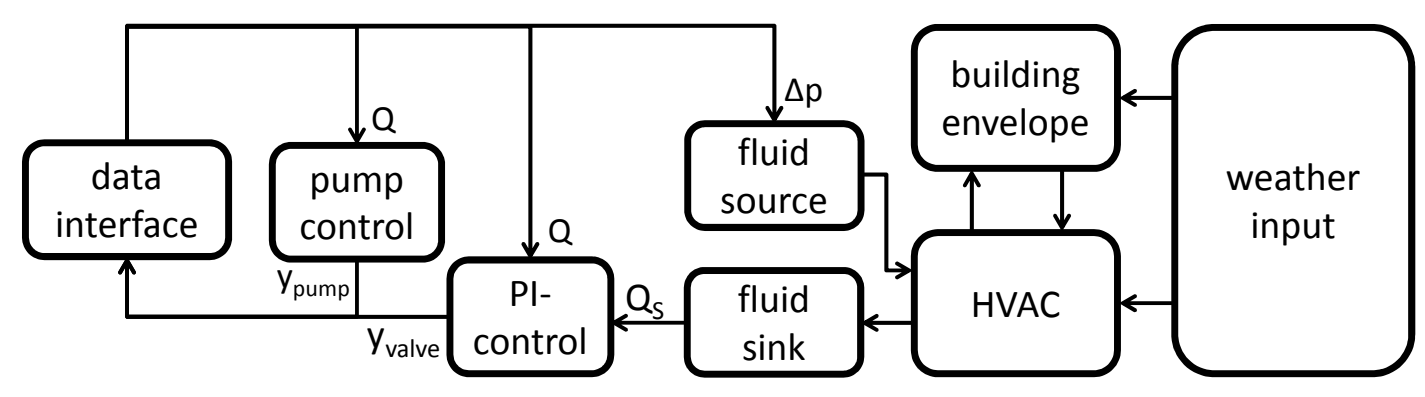

Figure 3. Scheme of the top level of a Modelica model for HIL-simulation, $\Delta \mathrm{p}$ - pressure difference, $\mathrm{Q}$ - volume flow rate in hydraulic circuit, $\mathrm{Q}_{\mathrm{S}}$ - simulated volume flow rate, $\mathrm{y}_{\text {pump }}$ - set point pump, $\mathrm{y}_{\text {valve }}$ - set point motor-driven valve. 
Modelica and compile the code with the Modelica code of the model.

Solutions for data interfaces for co-simulation and HIL already exist (e.g. Bellmann, 2009; Wetter, 2011b). We discovered in our application that the own implementation of a socket-based data interface allows a tailored design of the interface featuring a good integration within the proprietary LabVIEW-based software. The developed TCP\IP-based data interface allows exchanging data with sampling rates of up to $100 \mathrm{~ms}$ between the simulation and the LabVIEW-based testbench software. We assess the sampling rates of the data interface to be sufficient for the usage in the presented HILsystem.

\subsection{Hardware Level}

The proposed HIL-system is implemented in a real testbench in the labs of WILO SE (see Figure 6). The hardware level includes a real pump which is installed in a hydraulic circuit of a testbench. The testbench includes facilities to measure the pressure difference across the pump and the volume flow rate in the hydraulic circuit. It includes an adjustable valve to emulate the hydraulic boundary conditions of the simulated building. Both the simulation in Dymola and the LabVIEW-based software to control the facilities for data acquisition run on a desktop PC placed near the testbench. We use Dymola with default settings and its capability to synchronize with an external Dynamic Data Exchange (DDE) server for (soft) real-time simulation on a Windows 7 (64-bit) platform. The LabVIEW-based software is used to operate the data acquisition and switch unit as well as providing a proprietary interface to the low-level pump motor control.

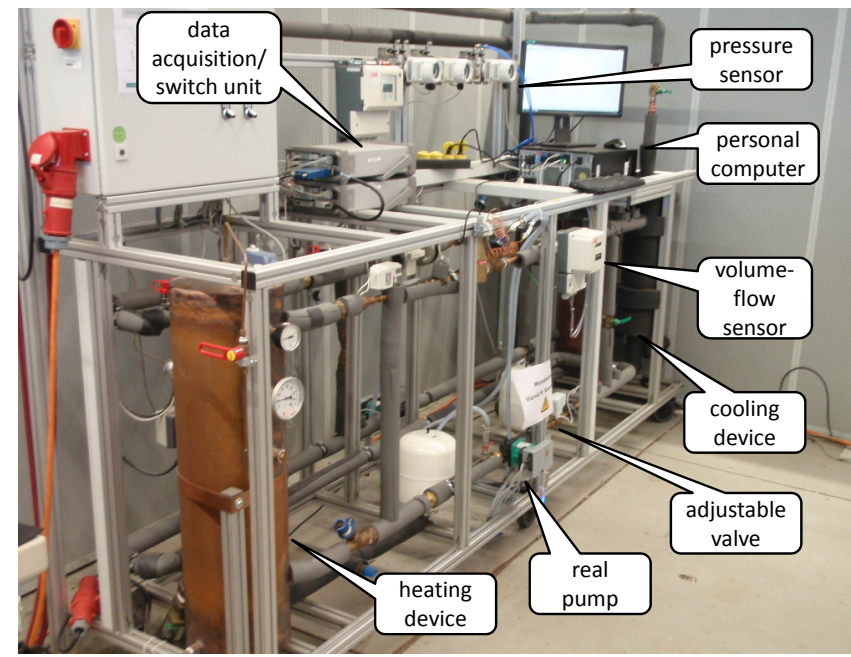

Figure 6. An implementation of the HIL-concept in a real testbench.

\section{Results}

To check the plausibility and evaluate the performance of the proposed HIL-setup for a circulating pump we present the results of a comparison between measured data from a HIL-simulation with calculated results of a full simulation in this section. Note, here HIL-simulation relates to a simulation of a building where the simulation is coupled to a real pump and full simulation relates to a simulation where the building is simulated including a model for a pump. In both cases we model a one family dwelling as described in section 3.1.

\subsection{HIL-Simulation}

In this section results for HIL-simulations are presented where a constant and a variable pressure difference control scheme are applied to control the pump. The set point of the pump head is set to $58.84 \mathrm{kPa}$ and $21.083 \mathrm{kPa}$ for the constant and variable pressure difference control scheme, respectively. In this specific test case the outside air temperature is set to $-12{ }^{\circ} \mathrm{C}$ and no solar radiation is considered. These specific boundary conditions are used to calculate the maximum needed power of the heating system. We model occupant actions by a ventilation schedule which assumes that the air is exchanged in every room once in the morning and once in the evening for half an hour. The rooms are ventilated separately with an offset of half an hour. The average air exchange by infiltration is assumed to be $0.5 \mathrm{~h}^{-1}$ over the whole day and during ventilation the thermostatic valves are closed. The set points of the room temperatures and basic parameters of the building model are reported in Table 1. A night control mode for the heating system is implemented which lowers the feed temperature of the heating medium between $10 \mathrm{pm}$ and $6 \mathrm{~m}$ from $75^{\circ} \mathrm{C}$ to $46{ }^{\circ} \mathrm{C}$. The described boundary conditions apply for both control schemes.

\section{Constant Pressure Difference Control Scheme}

The results for a real-time HIL-simulation of a constant pressure difference control scheme for one day are presented in Figure 7. The measured volume flow rate and pump head in the hydraulic circuit, $\mathrm{Q}_{\mathrm{m}}$ and $\mathrm{H}_{\mathrm{m}}$ and the corresponding simulated values $Q_{s}$ and $H_{s}$ of the full simulation are depicted. For better understanding the deviations of the volume flow rates the results of the simulated room temperature $\mathrm{T}_{\text {Room }}$ and the set temperature $\mathrm{T}_{\text {Set }}$ for the thermostatic valve of an exemplary room in the lower floor are presented in Figure 9.

Due to the set boundary conditions the volume flow rates $\mathrm{Q}_{\mathrm{m}}$ and $\mathrm{Q}_{\mathrm{s}}$ of the building vary between 100 and $800 \mathrm{l} / \mathrm{h}$ during the day. Gradients of about $7001 \mathrm{~h}^{2}$ occur when the thermostatic valves are reopened after the ventilation phases from 9am to $10 \mathrm{am}$ and $6 \mathrm{pm}$ to 8pm (see Figure 7 and 9). Oscillations of the head $\mathrm{H}_{\mathrm{m}}$ are caused by the PI-control yielding a feedback of the 

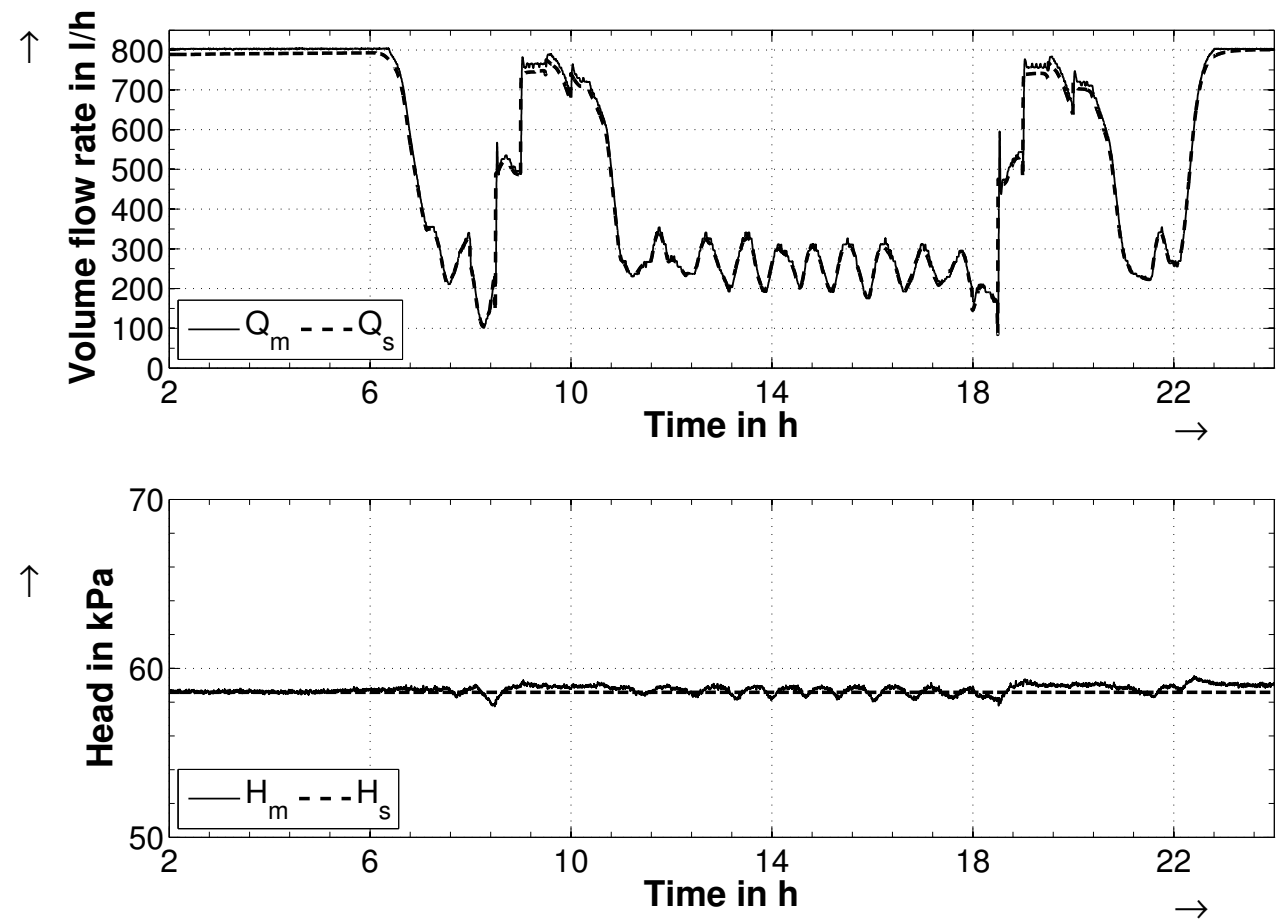

Figure 7. Comparison of a real-time HIL-simulation with full simulation for one day applying a constant pressure difference control scheme to the pump. $\mathrm{Q}_{\mathrm{m}}, \mathrm{H}_{\mathrm{m}}$ measured values for HIL-simulation, $\mathrm{Q}_{\mathrm{s}}, \mathrm{H}_{\mathrm{s}}$ simulated values for full simulation, volume flow rate and head of the pump, respectively, sampling rate: $1 \mathrm{~s}$.
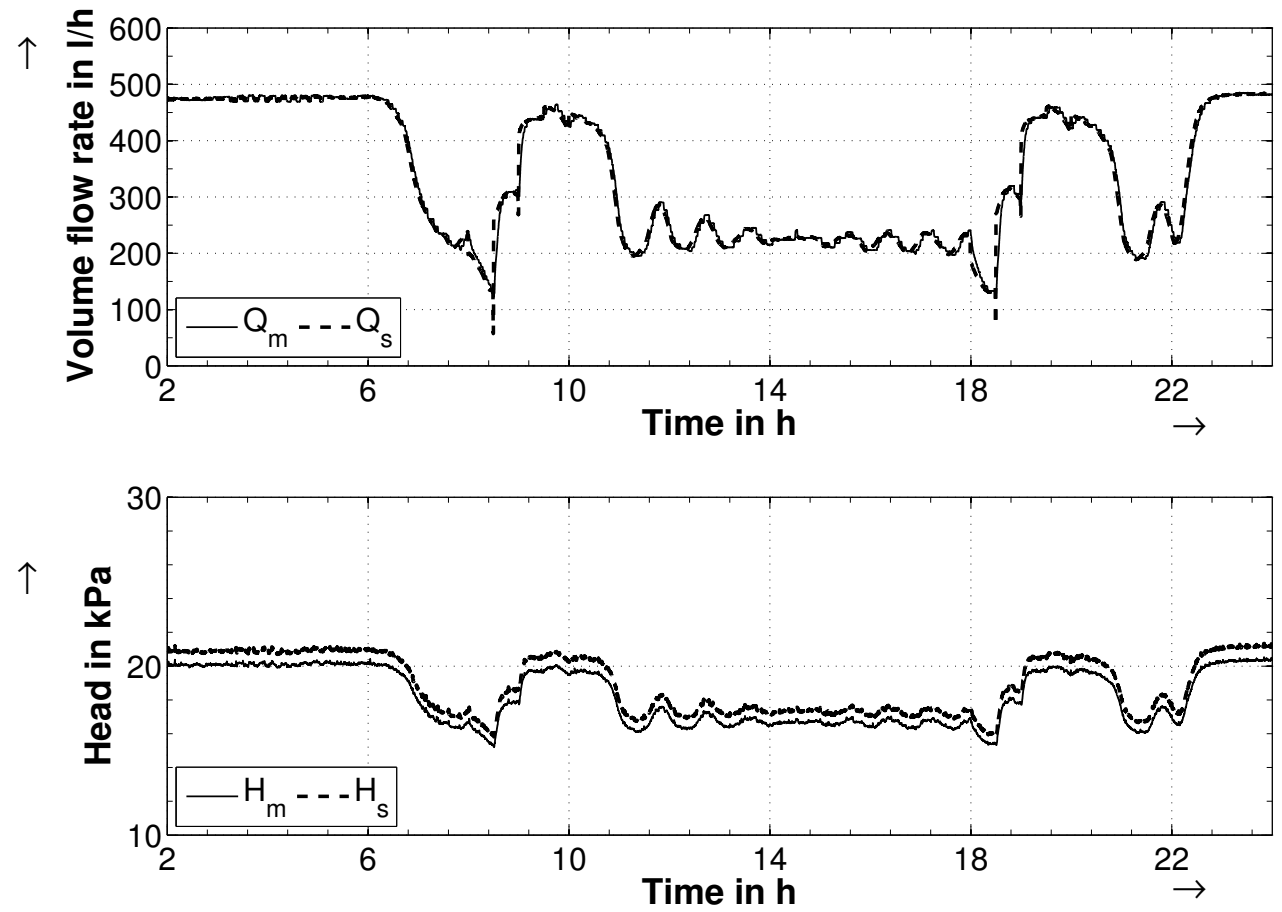

Figure 8. Comparison of a real-time HIL-simulation with full simulation for one day applying a variable pressure difference control scheme to the pump. $\mathrm{Q}_{\mathrm{m}}, \mathrm{H}_{\mathrm{m}}$ measured values for HIL-simulation, $\mathrm{Q}_{\mathrm{s}}, \mathrm{H}_{\mathrm{s}}$ simulated values for full simulation, volume flow rate and head of the pump, respectively, sampling rate: $1 \mathrm{~s}$. 
Table 1. Selected parameter of the building model of a one family dwelling according to energy saving ordinance from 1984. Design heat load calculated according to EN 12831 (CEN, 2003).

\begin{tabular}{ll}
\hline Parameter & Value \\
\hline Total length & $11.49 \mathrm{~m}$ \\
Total width & $8.74 \mathrm{~m}$ \\
Total area & $100.42 \mathrm{~m}^{2}$ \\
Room height & $2.6 \mathrm{~m}$ \\
Number of floors & 2 \\
Infiltration rate & $3 \mathrm{~h}^{-1}$ \\
Ventilation rate & $0.5 \mathrm{~h}^{-1}$ \\
Set temperature bathroom & $24^{\circ} \mathrm{C}$ \\
Set temperature hallway & $18{ }^{\circ} \mathrm{C}$ \\
Set temperature other rooms & $22^{\circ} \mathrm{C}$ \\
Design heat load & $8.97 \mathrm{~kW}$ \\
Design volume flow rate & $1.1 \mathrm{~m}^{3} / \mathrm{h}$ \\
Design head & $22.56 \mathrm{kPa}$ \\
Location & Mannheim/ Germany \\
\hline
\end{tabular}

volume flow rate oscillations to the pump head $\mathrm{H}_{\mathrm{m}}$.

The results provide evidence that the volume flow rate control system faces no difficulties in emulating the simulated gradients. To quantify the quality of the emulation we compare the measured and simulated values by calculating a root mean squared error and a relative mean error of $17.79 \mathrm{l} / \mathrm{h}(2.40 \%)$ and $307.92 \mathrm{~Pa}$ $(0.44 \%)$ for the volume flow rates and pump heads, respectively.

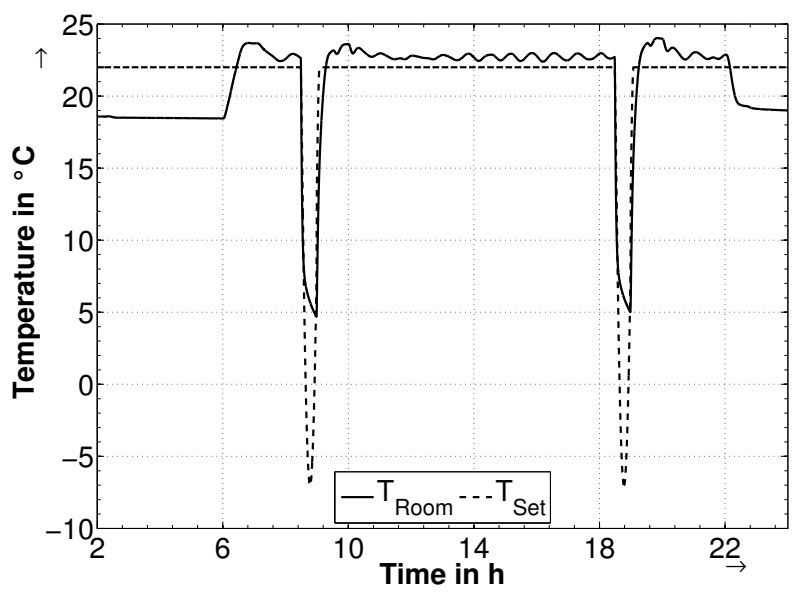

Figure 9. Simulated room temperature $T_{\text {Room }}$ and set temperature $\mathrm{T}_{\mathrm{Set}}$ of one room of the building for one day with constant pressure control scheme applied and sampling rate: $1 \mathrm{~s}$.

\section{Variable Pressure Difference Control Scheme}

For a variable pressure difference control scheme we present the results of a one day HIL-simulation in Figure 8. Volume flow rates and pump heads $\mathrm{Q}_{\mathrm{m}}$ and $\mathrm{H}_{\mathrm{m}}$ refer to the measured values in the hydraulic circuit of the testbench and $\mathrm{Q}_{\mathrm{s}}$ and $\mathrm{H}_{\mathrm{s}}$ to the simulated values for the full simulation, respectively. The corresponding results for the simulated room temperature $\mathrm{T}_{\text {Room }}$ and the set temperature $\mathrm{T}_{\text {Set }}$ for the thermostatic valve of an exemplary room in the lower floor are given in Figure 10. Temperature trajectories in Figure 9 and 10 are from the same room.

As the boundary conditions remain equal for the constant and the variable pressure difference control scheme, the trajectories of the volume flow rates $Q_{m}$ and $Q_{s}$ in Figure 7 and 8 show a similar pattern. Of interest are the pump head trajectories $\mathrm{H}_{\mathrm{m}}$ and $\mathrm{H}_{\mathrm{s}}$, shown in Figure 8, which are lowered from set point $21.08 \mathrm{kPa}$ to about $17.16 \mathrm{kPa}$ from $11 \mathrm{am}$ and $6 \mathrm{pm}$ where the volume flow rate and respectively the heating demand is low compared to the ventilation times (see Figure 10). While maintaining the thermal comfort by keeping the room temperature $\mathrm{T}_{\text {Room }}$ above the set temperature $\mathrm{T}_{\text {Set }}$ during day time (Figure 10), the variable pressure difference control scheme reduces the pump head. This yields a decrease of the amount of energy needed to operate the pump.

To compare the measured and simulated values we calculate a root mean squared error and a relative mean error of $14.49 \mathrm{l} / \mathrm{h}(3.04 \%)$ and $735.48 \mathrm{~Pa}(3.86 \%)$ for the volume flow rates and pump heads, respectively.

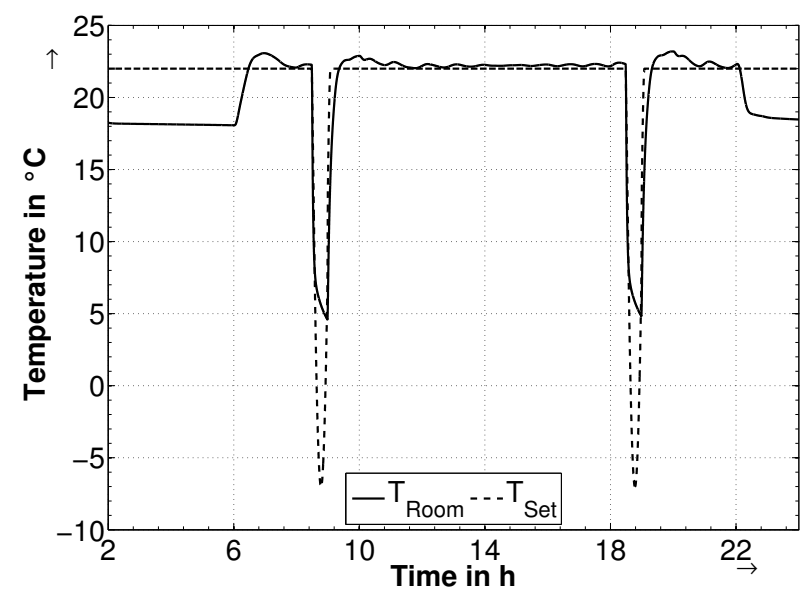

Figure 10. Simulated room temperature $\mathrm{T}_{\text {Room }}$ and set temperature $\mathrm{T}_{\mathrm{Set}}$ of one room of the building for one day with variable pressure control scheme applied and sampling rate: $1 \mathrm{~s}$.

\subsection{Discussion}

For HIL-simulation specific hardware exists which ensures simulation in hard real-time, e.g. by real-time capable operating systems. The HIL-simulations presented in this work have been conducted on a generic desktop PC running on Windows 7 using the described models (see section 3.1) without other than the reported adjustments. Our findings indicate that with sampling rates and feedback control in the range of seconds, soft real-time simulation is sufficient. This concurs with the findings 
presented by Gäfvert et al. (2008). An implication of this is that for the presented application no additional investments in the simulation infrastructure are necessary for HIL.

We assume that increasing the complexity of the models, e.g. investigate multiple-family dwellings, will not cause any problems since solutions times for one time step of the building model are well below the sampling time of the HIL-system. In our application the sampling time is limited to one second. This is related to the proprietary LabVIEW-based testbench software, mainly caused by a overhead generated from data handling algorithms in an integrated database.

The comparison of simulated results and measured data provide evidence that the solutions for the emulation of the hydraulic boundary conditions as well as the implemented data interface presented in this work show a sufficient performance for the investigated application with calculated mean relative errors less than $4 \%$.

Real hardware signals have a significant impact on the robustness of control algorithms. In Figure 7 simulated results for volume flow rate and pump head are continuous, whereas discrete data acquisition and measurement noise alter the measured signals. In contrast to the transition of model-based designed control algorithms which have not been tested on real hardware, HIL-simulation assisted transition offers the additional benefit to deduce improvements and increase the robustness of model-based designed algorithms as these signals are considered already during the design process and prior to commissioning. The quality of the emulation systems needs to be evaluated carefully to exclude an influence of these devices on the measurements.

HIL-simulation in the described context offers the benefit that, in contrast to measurements in real building energy and control systems, the boundary conditions of test scenarios can be chosen freely by the modeller and be reproduced almost equally on a testbench for multiple testing.

\section{Conclusion}

In this paper we present the application of the HILmethod to a building energy and control system to investigate circulating pump control. The presented work evaluates how HIL-simulation of building energy control systems using Modelica can be used to bridge the gap between design and commissioning stage of control algorithms for HVAC components, e.g. circulating pumps. We describe in detail the solutions found for the emulation of the hydraulic boundary conditions and a socketbased data interface. HIL-simulations are performed on an implementation of the concept. The quality of implementation is evaluated with results obtained from HILsimulations where a constant and a variable pressure control scheme are applied to the pump. For a comparison of measured data against simulated results we calculate a root mean squared error and a relative mean error of $17.79 \mathrm{l} / \mathrm{h}(2.40 \%)$ and $307.92 \mathrm{~Pa}(0.44 \%)$ for the volume flow rates and the pump heads applying a constant pressure difference control scheme, respectively. For results obtained by applying a variable pressure difference control scheme we calculate a root mean squared error and a relative mean error of $14.49 \mathrm{l} / \mathrm{h}(3.04 \%)$ and $735.48 \mathrm{~Pa}$ $(3.86 \%)$ for the volume flow rates and the pump heads, respectively.

With the HIL-system presented in this paper, obstacles of the transition from design to commissioning stage of circulating pump control are solved at once:

- Model-based designed algorithms are tested directly on real hardware without reimplementation in an application controller;

- The usage of object-oriented model libraries allows to investigate different types of buildings and HVAC systems by exchanging component models on one hardware setup.

If correctly applied cost and time savings are expected for manufacturers as the methodology significantly speeds up and simplifies the transition process. Additionally, the methodology offers the benefit of enabling the feedback of insights to the design process from running newly developed control algorithms on real hardware.

A possibility to accelerate the still required reimplementation of the developed algorithms is using automated code generation techniques. HIL-simulation is a additional tool in the transition process. Nevertheless, to complete product development thorough testing and a quality insurance process are still necessary.

Results presented in this paper partly rely on proprietary code especially for the HVAC-system models. A future development could comprise of an open source library providing ready-to-use models for HIL of thermal and hydraulic components of building energy and control systems.

The methodology of this work has been tested with models for one family dwelling with a limited complexity. Future work could focus on expanding to more complex building models in terms of the number of modelling equations.

\section{Acknowledgements}

This research is part of a master thesis which took place at WILO SE supervised by the Institute for Energy Efficient Buildings and Indoor Climate. We would like to thank WILO SE for financial support of the research activities. 


\section{References}

ASHRAE, 2004. Standard Method of Test for the Evaluation of Building Energy Analysis Computer Programs, July 2004. American Society of Heating, Refrigerating and Air-Conditioning Engineers, Atlanta, USA.

T. Bellmann. Interactive Simulations and Advanced Visualization with Modelica. In F. Casella, editor, Proceedings of the 7th Modelica Conference, pages 541-550. Linköping University Elektronic Press, Como, Italy, September 20-22 2009. doi:10.3384/ecp09430056.

M. Bonvini, F. Donida, and A. Leva. Modelica as a Design Tool for Hardware-in-the-Loop Simulation. In F. Casella, editor, Proceedings of the 7th International Modelica Conference, pages 378-385. Linköping University Elektronic Press, Como, Italy, September 20-22 2009. doi:10.3384/ecp09430119.

CEN, 2003. Heating Systems in Buildings - Method for Calculation of the Design Heat Load; German Version, DIN EN 12831, 2003. European Commitee for Standardization (CEN), Brussels, Belgium.

K. Chen, R. Streblow, D. Müller, A. Benigni, C. Molitor, and A. Monti. Hardware-in-the-Loop Simulationsverfahren für Hausenergiesysteme. In Proceedings of the Fourth GermanAustrian IBPSA Conference. IBPSA-Germany, Berlin University of the Arts, September 2012.

A. Constantin, R. Streblow, and D. Müller. The Modelica HouseModels Library: Presentation and Evaluation of a Room Model with the ASHRAE Standard 140. In H. Tummescheit and K.-E. Årzén, editors, Proceedings of the 10th International Modelica Conference, pages 293299. Linköping University Elektronic Press, Lund, Sweden, March 10-12 2014. doi:10.3384/ecp14096.

R. De Coninck, R. Baetens, D. Saelens, A. Woyte, and L. Helsen. Rule-Based Demand-Side Management of Domestic Hot Water Production With Heat Pumps in Zero Energy Neighbourhoods. Journal of Building Performance Simulation, 7(4):271-288, 2014. doi:10.1080/19401493.2013.801518.

DWD, 2013. Test reference year, Deutscher Wetter Dienst (DWD), Offenbach, Germany, http://www.dwd.de/TRY, Accessed: 25.04.2015.

$\begin{array}{lccc}\text { Dymola, } & 2013 . & \text { Dassault } & \text { Systemes AB, } \\ \text { Lund, } & \text { Sweden, } & \text { http://www.3ds.com/products- } \\ \text { services/catia/portfolio/dymola. }\end{array}$

EU, 2009. Directive 2009\125 \EC of the European Parliament and of the Council Establishing a Framework for the Setting of Eco-Design Requirements for Energy-Related Products, October 2009.

M. Gäfvert, T. Skoglung, H. Tummescheit, J. Windahl, H. Wikander, and P. Reuterswärd. Real-Time HWIL Simulation of Liquid Food Process Lines. In B. Bachmann, editor, Proceedings of the 6th International Modelica Conference, volume 2, pages 709-715. The Modelica Association and University of Applied Sciences Bielefeld, Bielefeld, Germany, March 3-4 2008.
D. Maclay. Simulation Gets Into the Loop. IEEE Review, 43 (3):109-112, May 1997.

Microsoft, 2015. Winsock library by Microsoft http://msdn.microsoft.com/enus/library/ms740673\%28VS.85\%29.aspx, Accessed: 15.04.2015.

D. Müller and A. Hosseini Badakhshani. Gekoppelte Gebäudeund Anlagensimulation mit Modelica. In Proceedings of the 3rd German-Austrian IBPSA Conference, September, 2224, Vienna, Austria 2010.

NI, 2015. LabVIEW, National Instruments, Austin, USA, http://www.ni.com/labview/.

Solar-Computer, 2012. SOLAR-COMPUTER GmbH, Göttingen, Germany, http://www.solar-computer.de.

M. Wetter. Building Performance Simulation for Design and Operation, chapter A View on Future Building System Modeling and Simulation, pages 1-28. Taylor \& Francis, 2011a. ISBN 978-0-415-47414-6.

M. Wetter. Co-Simulation of Building Energy and Control System with the Building Controls Virtual Test Bed. Journal of Building Performance Simulation, 4(3):185-203, $2011 \mathrm{~b}$. doi:10.1080/19401493.2010.518631.

M. Wetter, W. Zuo, T. S. Nouidui, and X. Pang. Modelica Buildings Library. Journal of Building Performance Simulation, 7(4):253-270, 2014. doi:10.1080/19401493.2013.765506.

D. Winkler and C. Gühmann. Hardware-in-the-Loop Simulation of a Hybrid Electric Vehicle Using Modelica/ Dymola. In Proceedings of the 22nd International Battery, Hybrid and Fuel Cell Electric Vehicle Symposium and Exhibition, pages 1054-1063, Yokohama, Japan, 2006.

P. Xu, P. Haves, and J. Deringer. A Simulation-Based Testing and Training Environment for Building Controls. In Proceedings of SimBuild 2004, Boulder, USA, 2004. 